\title{
Effects of Supernova Feedback on the Formation of Galaxies
}

\author{
Cecilia Scannapieco $^{1}$, Patricia B. Tissera ${ }^{2}$, Simon D. M. White ${ }^{1}$ \\ and Volker Springel ${ }^{1}$ \\ ${ }^{1}$ Max-Planck Institute for Astrophysics \\ Karl-Schwarzchild Str. 1, D85748, Garching, Germany \\ email: cecilia@mpa-garching.mpg.de \\ ${ }^{2}$ Instituto de Astronomía y Física del Espacio \\ Casilla de Correos 67, Suc. 28, 1428, Buenos Aires, Argentina
}

\begin{abstract}
We study the effects of Supernova (SN) feedback on the formation of galaxies using hydrodynamical simulations in a $\Lambda \mathrm{CDM}$ cosmology. We use an extended version of the code GADGET-2 which includes chemical enrichment and energy feedback by Type II and Type Ia $\mathrm{SN}$, metal-dependent cooling and a multiphase model for the gas component. We focus on the effects of SN feedback on the star formation process, galaxy morphology, evolution of the specific angular momentum and chemical properties. We find that SN feedback plays a fundamental role in galaxy evolution, producing a self-regulated cycle for star formation, preventing the early consumption of gas and allowing disks to form at late times. The SN feedback model is able to reproduce the expected dependence on virial mass, with less massive systems being more strongly affected.
\end{abstract}

Keywords. galaxies: formation, galaxies: evolution, methods: n-body simulations

\section{Introduction}

Supernova explosions play a fundamental role in galaxy formation and evolution. On one side, they are the main source of heavy elements in the Universe and the presence of such elements substantially enhances the cooling of gas (White \& Frenk 1991). On the other hand, SNe eject a significant amount of energy into the interstellar medium. It is believed that SN explosions are responsible of generating a self-regulated cycle for star formation through the heating and disruption of cold gas clouds, as well as of triggering important galactic winds such as those observed (e.g. Martin 2004). Smaller systems are more strongly affected by SN feedback, because their shallower potential wells are less efficient in retaining baryons (e.g. White \& Frenk 1991).

Numerical simulations have become an important tool to study galaxy formation, since they can track the joint evolution of dark matter and baryons in the context of a cosmological model. However, this has shown to be an extremely complex task, because of the need to cover a large dynamical range and describe, at the same time, largescale processes such as tidal interactions and mergers and small-scale processes related to stellar evolution.

One of the main problems that galaxy formation simulations have repeteadly found is the inability to reproduce the morphologies of disk galaxies observed in the Universe. This is generally refered to as the angular momentum problem that arises when baryons transfer most of their angular momentum to the dark matter components during interactions and mergers (Navarro \& Benz 1991; Navarro \& White 1994). As a result, disks are too small and concentrated with respect to real spirals. More recent simulations which include prescriptions for SN feedback have been able to produce more realistic disks 
(e.g. Abadi et al. 2003; Robertson et al. 2004; Governato et al. 2007). These works have pointed out the importance of SN feedback as a key process to prevent the loss of angular momentum, regulate the star formation activity and produce extended, young disk-like components.

In this work, we investigate the effects of SN feedback on the formation of galaxies, focusing on the formation of disks. For this purpose, we have run simulations of a Milky-Way type galaxy using an extended version of the code GADGET-2 which includes chemical enrichment and energy feedback by SN. A summary of the simulation code and the initial conditions is given in Section 2. In Section 3 we investigate the effects of SN feedback on galaxy morphology, star formation rates, evolution of specific angular momentum and chemical properties. We also investigate the dependence of the results on virial mass. Finally, in Section 4 we give our conclusions.

\section{Simulations}

We use the simulation code described in Scannapieco et al. (2005, 2006). This is an extended version of the Tree-PM SPH code GADGET-2 (Springel \& Hernquist 2002; Springel 2005), which includes chemical enrichment and energy feedback by SN, metaldependent cooling and a multiphase model for the gas component. Note that our star formation and feedback model is substantially different from that of Springel \& Hernquist (2003), but we do include their treatment of UV background.

We focus on the study of a disk galaxy similar to the Milky Way in its cosmological context. For this purpose we simulate a system with $z=0$ halo mass of $\sim 10^{12} h^{-1}$ $\mathrm{M}_{\odot}$ and spin parameter of $\lambda \sim 0.03$, extracted from a large cosmological simulation and resimulated with improved resolution. It was selected to have no major mergers since $z=1$ in order to give time for a disk to form. The simulations adopt a $\Lambda \mathrm{CDM}$ Universe with the following cosmological parameters: $\Omega_{\Lambda}=0.7, \Omega_{\mathrm{m}}=0.3, \Omega_{\mathrm{b}}=0.04$, a normalization of the power spectrum of $\sigma_{8}=0.9$ and $H_{0}=100 \mathrm{hm} \mathrm{s}^{-1} \mathrm{Mpc}^{-1}$ with $h=0.7$. The particle mass is $1.6 \times 10^{7}$ for dark matter and $2.4 \times 10^{6} h^{-1} \mathrm{M}_{\odot}$ for baryonic particles, and we use a maximum gravitational softening of $0.8 h^{-1} \mathrm{kpc}$ for gas, dark matter and star particles. At $z=0$ the halo of our galaxy contains $\sim 1.2 \times 10^{5}$ dark matter and $\sim 1.5 \times 10^{5}$ baryonic particles within the virial radius.

In order to investigate the effects of SN feedback on the formation of galaxies, we compare two simulations which only differ in the inclusion of the SN energy feedback model. These simulations are part of the series analysed in Scannapieco et al. (2008), where an extensive investigation of the effects of SN feedback on galaxies and a parameter study is performed. In this work, we use the no-feedback run NF (run without including the SN energy feedback model) and the feedback run E-0.7. We refer the interested reader to Scannapieco et al. (2008) for details in the characteristics of these simulations.

\section{Results}

In Fig. 1 we show stellar surface density maps at $z=0$ for the NF and E- 0.7 runs. Clearly, SN feedback has an important effect on the final morphology of the galaxy. If $\mathrm{SN}$ feedback is not included, as we have done in run NF, the stars define a spheroidal component with no disk. On the contrary, the inclusion of SN energy feedback allows the formation of an extended disk component.

The generation of a disk component is closely related to the star formation process. In the left-hand panel of Fig. 2 we show the star formation rates (SFR) for our simulations. In the no-feedback case (NF), the gas cools down and concentrates at the centre of the 

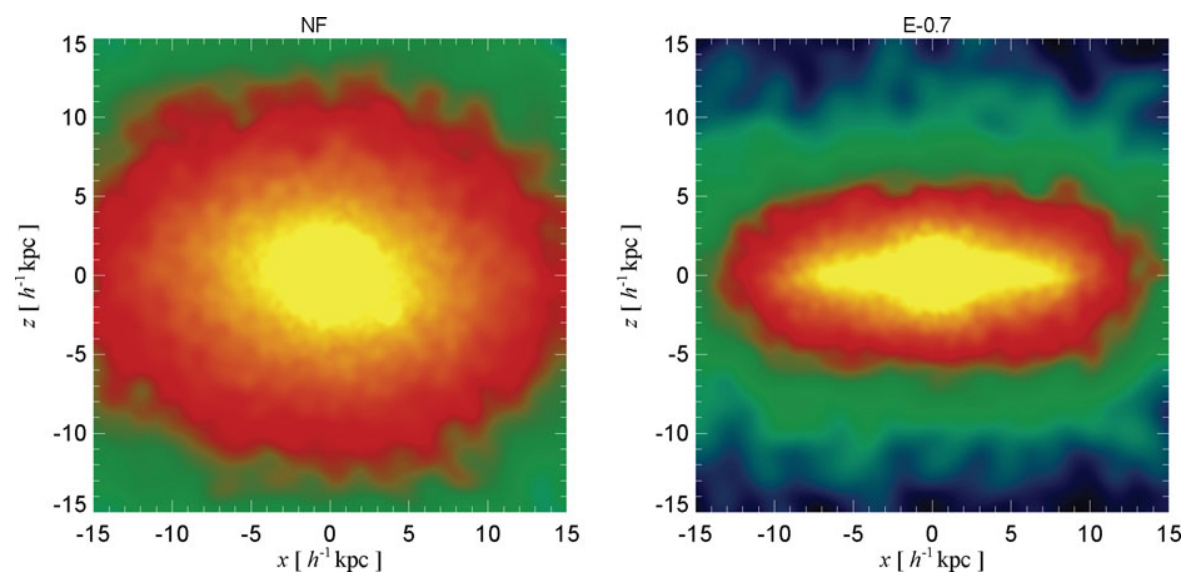

Figure 1. Edge-on stellar surface density maps for the no-feedback (NF, left-hand panel) and feedback (E-0.7, right-hand panel) simulations at $z=0$. The colors span 4 orders of magnitude in projected density, with brighter colors representing higher densities.
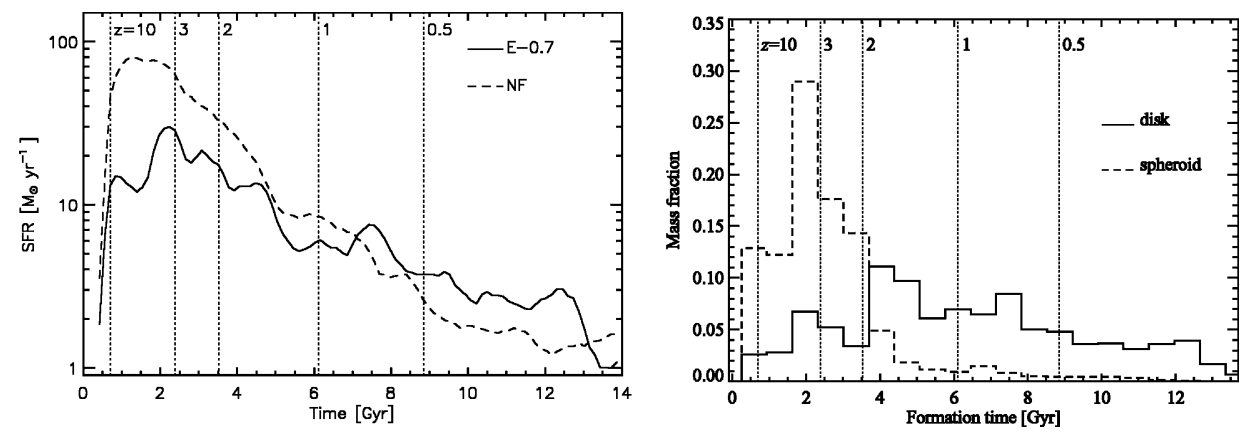

Figure 2. Left: Star formation rates for the no-feedback (NF) and feedback (E-0.7) runs. Right: Mass fraction as a function of formation time for stars of the disk and spheroidal components in simulation E-0.7.

potential well very early, producing a strong starburst which feeds the galaxy spheroid. As a result of the early consumption of gas to form stars, the SFR is low at later times. On the contrary, the SFR obtained for the feedback case is lower at early times, indicating that SN feedback has contributed to self-regulate the star formation process. This is the result of the heating of gas and the generation of galactic winds. In this case, the amount of gas available for star formation is larger at recent times and consequently the SFR is higher. In the right-hand panel of Fig. 2 we show the mass fraction as a function of formation time for stars of the disk and spheroidal components in our feedback simulation (see Scannapieco et al. 2008 for the method used to segregate stars into disk and spheroid). ¿From this plot it is clear that star formation at recent times $(z \lesssim 1)$ significantly contributes to the formation of the disk component, while stars formed at recent times contribute mainly to the spheroid. In this simulation, $\sim 50$ per cent of the mass of the disk forms since $z=1$. Note that in the no-feedback case, only a few per cent of the final stellar mass of the galaxy is formed since $z=1$.

Our simulation E-0.7 has produced a galaxy with an extended disk component. By using the segregation of stars into disk and spheroid mentioned above, we can calculate the masses of the different components, as well as characteristic scales. The disk of the 

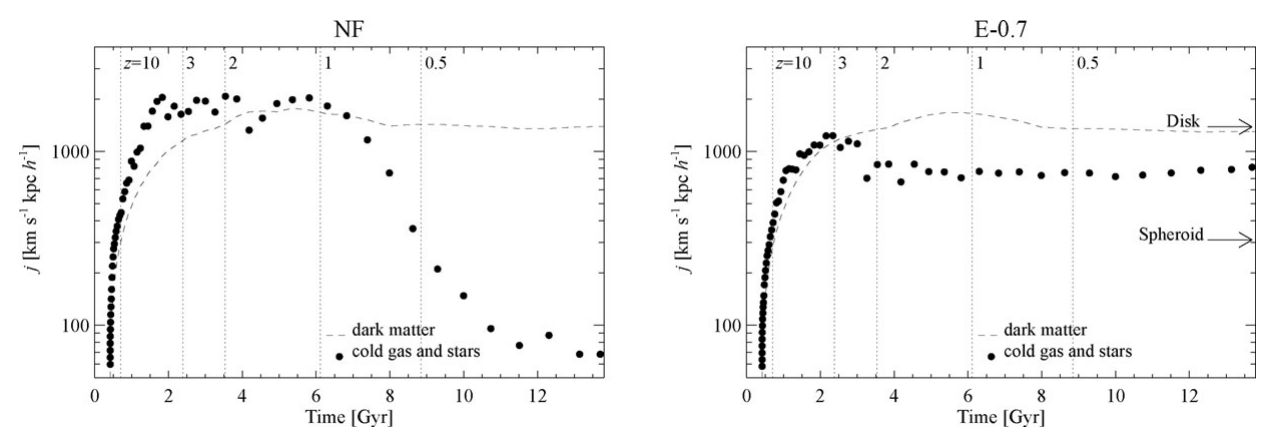

Figure 3. Dashed lines show the specific angular momentum as a function of time for the dark matter that, at $z=0$, lies within the virial radius of the system for NF (left panel) and E- 0.7 (right panel). We also show with dots the specific angular momentum for the baryons which end up as cold gas or stars in the central $20 h^{-1} \mathrm{kpc}$ at $z=0$. The arrows show the specific angular momentum of disk and spheroid stars.

simulated galaxy has a mass of $3.3 \times 10^{10} h^{-1} M_{\odot}$, a half-mass radius of $5.7 h^{-1} \mathrm{kpc}$, a half-mass height of $0.5 h^{-1} \mathrm{kpc}$, and a half-mass formation time of $6.3 \mathrm{Gyr}$. The spheroid mass and half-mass formation time are $4.1 \times 10^{10} h^{-1} M_{\odot}$ and $2.5 \mathrm{Gyr}$, respectively. It is clear that the characteristic half-mass times are very different in the two cases, the disk component being formed by younger stars.

In Fig. 3 we show the evolution of the specific angular momentum of the dark matter (within the virial radius) and of the cold gas plus stars (within twice the optical radius) for the no-feedback case (left-hand panel) and for the feedback case E-0.7 (right-hand panel). The evolution of the specific angular momentum of the dark matter component is similar in the two cases, growing as a result of tidal torques at early epochs and being conserved from turnaround $(z \approx 1.5)$ until $z=0$. On the contrary, the cold baryonic components in the two cases differ significantly, in particular at late times. In the no-feedback case (NF), much angular momentum is lost through dynamical friction, particularly through a satellite which is accreted onto the main halo at $z \sim 1$. In E-0.7, on the other hand, the cold gas and stars lose rather little specific angular momentum between $z=1$ and $z=0$. Two main factors contribute to this difference. Firstly, in E-0.7 a significant number of young stars form between $z=1$ and $z=0$ with high specific angular momentum (these stars form from high specific angular momentum gas which becomes cold at late times); and secondly, dynamical friction affects the system much less than in NF, since satellites are less massive. At $z=0$, disk stars have a specific angular momentum comparable to that of the dark matter, while spheroid stars have a much lower specific angular momentum.

In Fig 4 we show the oxygen profiles for the no-feedback (NF) and feedback (E-0.7) runs. ¿From this figure we can see that SN feedback strongly affects the chemical distributions. If no feedback is included, the gas is enriched only in the very central regions. Including SN feedback triggers a redistribution of mass and metals through galactic winds and fountains, giving the gas component a much higher level of enrichment out to large radii. A linear fit to this metallicity profile gives a slope of $-0.048 \mathrm{dex} \mathrm{kpc}^{-1}$ and a zero-point of 8.77 dex, consistent with the observed values in real disk galaxies (e.g. Zaritsky et al. 1994).

Finally, we investigate the effects of SN feedback on different mass systems. For that purpose we have scaled down our initial conditions to generate galaxies of $10^{10} h^{-1} M_{\odot}$ and $10^{9} h^{-1} M_{\odot}$ halo mass, and simulate their evolution including the SN feedback 


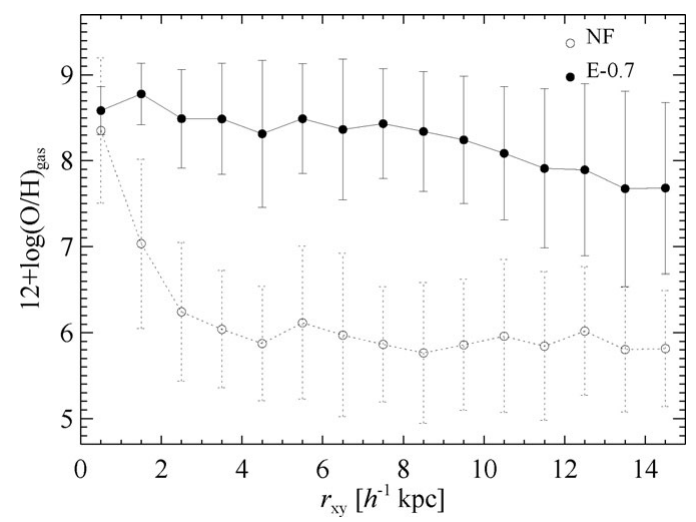

Figure 4. Oxygen abundance for the gas component as a function of radius projected onto the disk plane for our no-feedback simulation (NF) and for the feedback case E-0.7. The error bars correspond to the standard deviation around the mean.

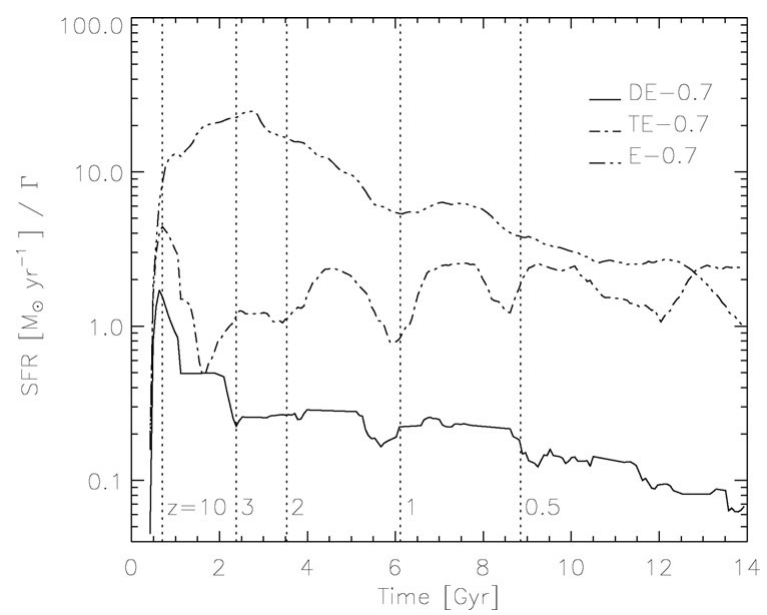

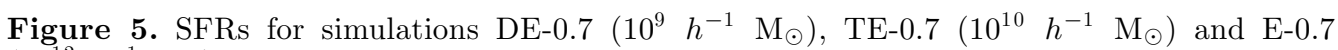
$\left(10^{12} h^{-1} \mathrm{M}_{\odot}\right)$ run with energy feedback. To facilitate comparison, the SFRs are normalized to the scale factor $\Gamma$.

model (with the same parameters than E-0.7). These simulations are TE-0.7 and DE0.7, respectively. In Fig. 5 we show the SFRs for these simulations, as well as for E-0.7, normalized to the scale factor $\left(\Gamma=1\right.$ for E- $0.7, \Gamma=10^{-2}$ for $T E-0.7$ and $\Gamma=10^{-3}$ for DE-0.7). From this figure it is clear that SN feedback has a dramatic effect on small galaxies. This is because more violent winds develop and baryons are unable to condensate and form stars. In the smallest galaxy, the SFR is very low at all times because most of the gas has been lost after the first starburst episode. This proves that our model is able to reproduce the expected dependence of SN feedback on virial mass, without changing the relevant physical parameters.

\section{Conclusions}

We have run simulations of a Milky Way-type galaxy in its cosmological setting in order to investigate the effects of $\mathrm{SN}$ feedback on the formation of galaxy disks. We compare two simulations with the only difference being the inclusion of the SN energy 
feedback model of Scannapieco et al. (2005, 2006). Our main results can be summarized as follows:

- SN feedback helps to settle a self-regulated cycle for star formation in galaxies, through the heating and disruption of cold gas and the generation of galactic winds. The regulation of star formation allows gas to be mantained in a hot halo which can condensate at late times, becoming a reservoir for recent star formation. This contributes significantly to the formation of disk components.

- When SN feedback is included, the specific angular momentum of the baryons is conserved and disks with the correct scale-lengths are obtained. This results from the late collapse of gas with high angular momentum, which becomes available to form stars at later times, when the system does not suffer from strong interactions.

- The injection of SN energy into the interstellar medium generates a redistribution of chemical elements in galaxies. If energy feedback is not considered, only the very central regions were stars are formed are contaminated. On the contrary, the inclusion of feedback triggers a redistribution of metals since gas is heated and expands, contaminating the outer regions of galaxies. In this case, metallicity profiles in agreement with observations are produced.

- Our model is able to reproduce the expected dependence of SN feedback on virial mass: as we go to less massive systems, SN feedback has stronger effects: the star formation rates (normalized to mass) are lower, and more violent winds develop. This proves that our model is well suited for studying the cosmological growth of structure where large systems are assembled through mergers of smaller substructures and systems form simultaneously over a wide range of scales.

\section{References}

Abadi, M. G., Navarro, J. F., Steinmetz, M., \& Eke, V. R., 2003, ApJ, 591, 499

Governato, F., Willman, B., Mayer, L., Brooks, A., Stinson, G., Valenzuela, O., Wadsley, J., \& Quinn, T., 2007, MNRAS, 374, 1479

Martin, C. L., 2004, $A \& A S, 205,8901$

Navarro, J. F. \& Benz, W., 1991, ApJ, 380, 320

Navarro, J. F. \& White, S. D. M., 1993, MNRAS, 265, 271

Robertson, B., Yoshida, N., Springel, V., \& Hernquist, L., 2004, ApJ, 606, 32

Scannapieco, C., Tissera, P. B., White, S. D. M., \& Springel, V., 2005, MNRAS, 364, 552

Scannapieco, C., Tissera, P. B., White, S. D. M., \& Springel, V., 2006, MNRAS, 371, 1125

Scannapieco, C., Tissera, P. B., White, S. D. M., \& Springel, V., 2008, MNRAS, in press (astro$\mathrm{ph} / 0804.3795)$

Springel, V. \& Hernquist, L., 2002, MNRAS, 333, 649

Springel, V. \& Hernquist, L., 2003, MNRAS, 339, 289

Springel, V. 2005, MNRAS, 364, 1105

White, S. D. M. \& Frenk, C. S., 1991, ApJ, 379, 52

Zaritsky, D., Kennicutt, R. C. Jr., \& Huchra, J. P., 1994, ApJ, 420, 87 